\title{
Note on the Asymptotic Expansion of the Mod- ified Bessel Function of the Second Kind
}

\author{
By E. Dempsey and G. C. Benson
}

Tables of modified Bessel functions of the second kind, $K_{n}(z)$, for integral order $n$ and for special forms of real positive argument $z$ have recently been computed [1]. To obtain the desired accuracy throughout the tables it was found necessary to look into the methods available for approximating the remainder term $R_{p}$ in the asymptotic expansion of the function:

$$
K_{n}(z)=\sum_{m=0}^{p-1} u_{m}+\left(R_{p} / u_{p}\right) u_{p}
$$

where

$$
u_{m}=\left(\frac{\pi}{2 z}\right)^{\frac{1}{2}} \frac{\Gamma\left(n+m+\frac{1}{2}\right)}{\Gamma\left(n-m+\frac{1}{2}\right)} \frac{e^{-z}}{m !(2 z)^{m}}
$$

$u_{p-1}$ is usually the least term (in absolute value) of the series. Since the converging factor $R_{p} / u_{p}$ may itself be developed as an asymptotic series, it is convenient to discuss this quantity rather than the remainder term.

In a treatment due to Dingle a general theory of asymptotic expansions and their converging factors [2] is applied to the hypergeometric function [3], special cases of which are the modified Bessel functions of the first and second kinds. The formula relevant to the latter case is as follows

$$
\frac{R_{p}}{u_{p}}=\sum_{t=0}^{\infty} \frac{\Gamma\left(n+\frac{1}{2}\right) \Gamma\left(n+p+\frac{1}{2}-t\right)}{\Gamma\left(n+\frac{1}{2}-t\right) \Gamma\left(n+p+\frac{1}{2}\right)}(-2 z)^{t} \Lambda_{p-n-\frac{1}{2}}^{(t)}(2 z)
$$

in which $u_{p}$ is now not necessarily the first term after the least term of the series in Eq (1) but may be a term some way beyond this. Going to the fifth term in Eq (3) the functions $\Lambda_{p-n-\frac{1}{2}}^{(t)}(2 z)$ are defined by

$$
\begin{aligned}
& \Lambda_{s}^{(0)}(s+\theta)=\frac{1}{2}-\frac{1}{8 s}(1-2 \theta)+\frac{1}{32 s^{2}}\left(1-2 \theta-4 \theta^{2}\right) \\
& \quad+\frac{1}{128 s^{3}}\left(1+6 \theta+8 \theta^{2}+8 \theta^{3}\right)-\frac{1}{512 s^{4}}\left(13+22 \theta+20 \theta^{2}+16 \theta^{3}+16 \theta^{4}\right)
\end{aligned}
$$

$$
\Lambda_{s}^{(1)}(s+\theta)=\frac{1}{4 s}-\frac{1}{16 s^{2}}(1+4 \theta)+\frac{1}{64 s^{3}}\left(3+8 \theta+12 \theta^{2}\right)
$$

$$
-\frac{1}{256 s^{4}}\left(11+20 \theta+24 \theta^{2}+32 \theta^{3}\right)
$$

$$
\Lambda_{s}^{(2)}(s+\theta)=-\frac{1}{8 s^{2}}+\frac{1}{16 s^{3}}(1+3 \theta)-\frac{1}{128 s^{4}}\left(5+12 \theta+24 \theta^{2}\right)
$$

Received November 13, 1959; in revised form, February 3, 1960. Issued as N.R.C. No. 5711.

* The coefficient of $\left(64 s^{3}\right)^{-1}$ in $\Lambda_{8}^{(1)}$ given in ref. [3] is incorrect; the correct form is given above. 
(7) $\Lambda_{8}^{(3)}(s+\theta)=\frac{1}{16 s^{3}}-\frac{1}{32 s^{4}}(1+4 \theta)$

(8) $\Lambda_{s}^{(4)}(s+\theta)=-\frac{1}{32 s^{4}}$

These equations are derived on the basis that $\theta$ is small. $\theta$ and $p$ are obtained from the relationships

$$
\begin{aligned}
p-n-\frac{1}{2} & =s \\
2 z & =s+\theta
\end{aligned}
$$

By restricting the value of $\theta$ to $|\theta| \leqq 0.5, s$ is forced to take on the half integral value nearest to $2 z$, since both $p$ and $n$ must be integral. Using this method the best results were obtained by calculating $K_{2}(z)$ and $K_{3}(z)$ and using the recursion formula

$$
K_{n+1}(z)=K_{n-1}(z)+\frac{2 n}{z} K_{n}(z)
$$

to calculate the $K_{n}(z)$ required. In the program for calculating $K_{n}(z)$ a convergence test was applied to each term of the series in Eq (3); the summation was terminated at the least term and, since the terms alternate in sign, one half of the following term was added to the sum.

An alternative treatment due to Burnett [4] starts from a representation of $R_{p} / u_{p}$ as a double integral which is subsequently reduced to a series of products of gamma and hypergeometric functions. Expanding this series leads to the following expression for the converging factor, which in this case is applied to the term following the least term of the series for $K_{n}(z)$

$$
\begin{array}{r}
\frac{R_{p}}{u_{p}}=\frac{1}{2}+\frac{1}{8 z}[\sigma-n+1]+\frac{1}{32 z^{2}}\left[\left(\frac{1}{2}-n\right)(2 \sigma-3 n)+\sigma^{2}+\frac{1}{2} \sigma-\frac{1}{4}\right] \\
+\frac{1}{128 z^{3}}\left[\left(\frac{1}{2}-n\right)\left(3 \sigma^{2}-11 \sigma n+9 n^{2}-\frac{5}{2} \sigma-\frac{5}{2}\right)+\sigma^{3}-\frac{5}{4} \sigma-\frac{1}{8}\right] \\
+\frac{1}{512 z^{4}}\left[( \frac { 1 } { 2 } - n ) \left(4 \sigma^{3}-28 \sigma^{2} n+48 \sigma n^{2}-27 n^{3}-11 \sigma^{2}+11 \sigma n-\frac{5}{2} n^{2}\right.\right. \\
\left.\left.-11 \sigma+16 n+\frac{27}{8}\right)+\sigma^{4}-\sigma^{3}-\frac{11}{4} \sigma^{2}+\frac{7}{8} \sigma+\frac{13}{16}\right]
\end{array}
$$

where

$$
\sigma=2 z-p+n-\frac{1}{2}
$$

Previously published versions of this series [4], [5] go only as far as the term in $z^{-2}$. It is easy to show that the successive powers of $z^{-1}$ given in Eq (12) may be derived completely from the first five terms of Eq (3); in other words, to the extent that the equations appropriate to them have been given, the two methods should be roughly 


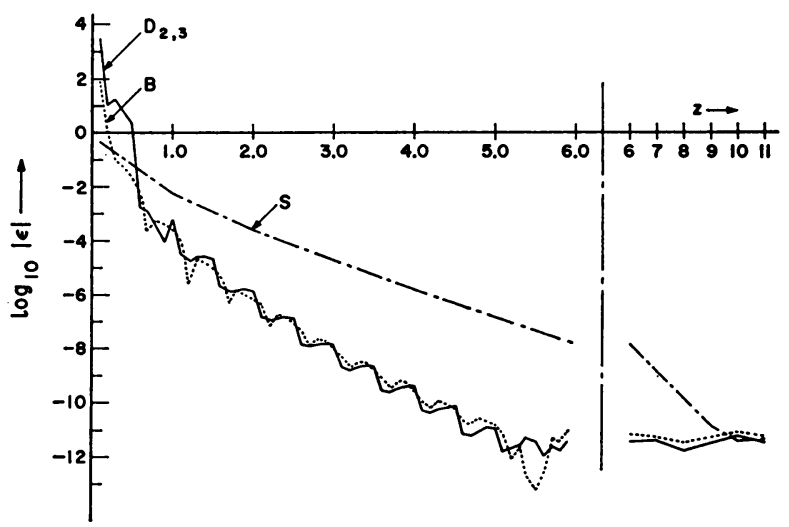

FIG. 1.-Deviation plot for calculations of $K_{0}(z)$

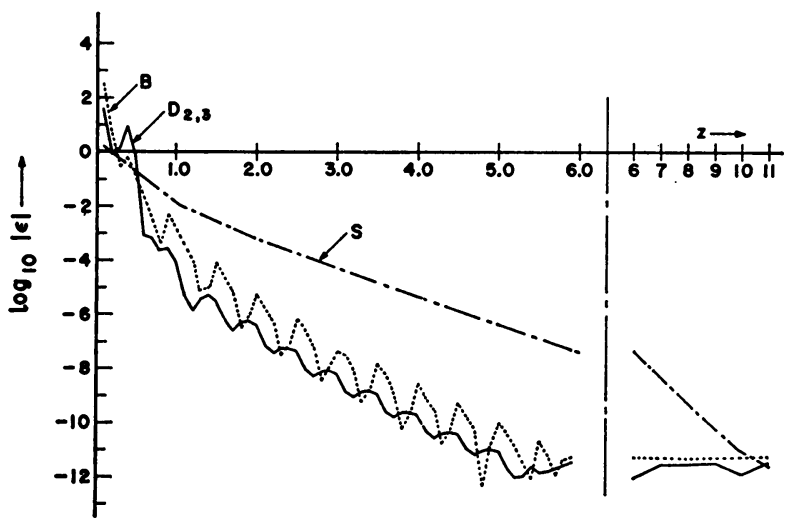

FIG. 2.-Deviation plot for calculations of $K_{1}(z)$

equivalent. In applying the Burnett treatment the converging factors for $K_{0}$ and $K_{1}$ were calculated from $\mathrm{Eq}$ (12) and functions of higher order obtained by use of the recursion formula.

The accompanying figures show graphs of $\log _{10}|\epsilon|$ against the argument $z$ for $K_{0}(z)$ and $K_{1}(z)$ calculated using the Dingle $\left(D_{2,3}\right)$ and Burnett (B) methods, where

$$
\epsilon=\frac{K(\text { exact })-K(\text { calc. })}{K(\text { exact })}
$$

The values for $K$ (exact) were taken from a paper by Aldis [6] which gives values of $K_{0}(z)$ and $K_{1}(z)$ in general accurate to more places than are required here. Curves $S$ were obtained using $\mathrm{Eq}(1)$ with $R_{p} / u_{p}$ set equal to 0.5 . The parts of the curves below about $|\epsilon|=10^{-11}$ should be ignored since they are influenced by the fact that the computer (a Bendix G15D) carried only twelve figures in the double precision floating point mode used.

It is apparent from Figures 1 and 2 that the Dingle method is somewhat better than that of Burnett. This may be due in part to the fact that the Dingle equations, as given here, contain an infinite number of terms which may be looked on as being 
incomplete Burnett type coefficients of powers of $z^{-1}$ higher than the fourth. Above about $z=10$ it is also evident that for a computer carrying only twelve figures there is nothing to be gained in using a more elaborate converging factor than $R_{p} / u_{p}=0.5$.

National Research Council of Canada Ottawa, Canada

1. E. Dempsey \& G. C. Benson, "Tables of the modified Bessel functions of the second kind for particular types of argument," Can Jn. Phys., v. 38, 1960, p. 399. This paper contains tables of $K_{n}\left(\frac{\pi}{2} \sqrt{q}\right)$ for $q=1(1.0) 250$ and of $K_{n}\left(\frac{\pi}{3} \sqrt{q}\right)$ for $q=1(1.0) 300$. In both cases values for integral orders 0 to 10 were computed to ten significant figures.

2. R. B. DINGLE, "Asymptotic expansions and converging factors. I. General theory and basic converging factors" "Proc., Roy. Soc., London, v. 244A, 1958, p. 456.

3. R. B. DingLe, "Asymptotic expansions and converging factors. IV Confluent hypergeometric, parabolic cylinder, modified Bessel, and ordinary Bessel functions," Proc., Roy. Soc., London, v. 249A, 1959, p. 270.

4. D. BURNETT, "The remainders in the asymptotic expansions of certain Bessel functions," Proc., Camb. Phil. Soc., v. 26, 1930, p. 145. p. 138

5. E. JAhNKE \& F. EmDE, Tables of Functions, Fourth Edition, Dover, New York, 1945,

6. W. S. ALDIs, "Tables for the solution of the equation $\frac{d^{2} y}{d x^{2}}+\frac{1}{x} \cdot \frac{d y}{d x}-\left(1+\frac{n^{2}}{x^{2}}\right) y=0$," Proc., Roy. Soc., London, v. 64, 1899, p. 203.

\section{On the Factors of Certain Mersenne Numbers}

\section{By John Brillhart and G. D. Johnson}

1. Introduction. For the past 10 months the authors have been conducting a search for factors of certain Mersenne numbers on the IBM 701 at the Computer Center, University of California, Berkeley. The following is a report on the nature and results of that search.

2. Extent. Prime factors $q$ were sought for the numbers $M_{p}=2^{p}-1$ for primes $p<1200$ in the intervals indicated:

$$
\begin{array}{lrl}
p=101 & & 2^{30}<q<2^{35} \\
103 \leqq p \leqq 157, & p \neq 151 & 2^{30}<q<2^{31} \\
157<p \leqq 257 & & 1<q<2^{31} \\
257<p \leqq 1021, & p \neq 397 & 1<q<2^{30} \\
p=397 & & 1<q<2^{32} \\
1021<p<1200 & 1<q<2^{28}
\end{array}
$$

No factors $<2^{30}$ were examined for $101 \leqq p \leqq 157$, since these had already been investigated [1]. No $M_{p}$ were examined for $p<101$ or $p=151$, since these numbers have presumably been completely factored. Possible factors $<2^{35}$ were

Received May 5, 1960. 